\title{
Expression and prognostic value of CLIC1 in epithelial ovarian cancer
}

\author{
WENTAO YU ${ }^{1,2}$, RAN CUI $^{1}$, HONG QU $^{1}$, CHONGDONG LIU $^{1}$, HAITENG DENG $^{3}$ and ZHENYU ZHANG ${ }^{1}$ \\ ${ }^{1}$ Department of Obstetrics and Gynecology, Beijing Chao-Yang Hospital, Capital Medical University, \\ Beijing 100020, P.R. China; ${ }^{2}$ Department of Vascular Surgery, Brigham and Women's Hospital, \\ Harvard Medical University, Boston, MA 02115, USA; ${ }^{3}$ MOE Key Laboratory of Bioinformatics, \\ School of Life Sciences, Tsinghua University, Beijing 100084, P.R. China
}

Received November 16, 2017; Accepted March 14, 2018

DOI: $10.3892 /$ etm.2018.6000

\begin{abstract}
The clinical significance of the chloride intracellular channel 1 (CLIC1) protein in ovarian cancer is yet to be determined. The present study aimed to investigate the association between CLIC1 expression, and clinicopathological features and prognosis of patients with epithelial ovarian cancer. In this retrospective study, CLIC1 level was determined by reverse transcription-quantitative polymerase chain reaction, western blotting and immunohistochemical staining. The association between CLIC1 expression and clinicopathological characteristics were evaluated. Progression-free survival and overall survival were assessed by univariate, and multivariate analyses. mRNA and protein levels of CLIC1 were significantly higher in cancerous tissues than in healthy ovarian tissues $(\mathrm{P}<0.001)$. CLIC1 signals in epithelial ovarian cancer tissues were significantly higher than that in healthy tissues $(\mathrm{P}<0.001)$. CLIC1 expression was significantly higher in higher-grade tumors than in low-grade tumors $(\mathrm{P}<0.001)$. Moreover, overexpression of CLIC1 was associated with cisplatin resistance $(\mathrm{P}<0.001)$. CLIC1 expression was an independent factor that predicted shorter progression-free survival $(\mathrm{P}=0.006)$ and overall survival $(\mathrm{P}=0.002)$ for patients with epithelial ovarian cancer. These findings indicate that CLIC1 is overexpressed and is associated with poor prognosis in patients with epithelial ovarian cancer.
\end{abstract}

Correspondence to: Dr Zhenyu Zhang, Department of Obstetrics and Gynecology, Beijing Chao-Yang Hospital, Capital Medical University, 8 Gongti South Street, Beijing 100020, P.R. China

E-mail: zhenyuzhang2000@163.com

Abbreviations: CA 125, cancer antigen 125; CLIC1, chloride intracellular channel 1; FIGO, International Federation of Gynecology and Obstetrics; OS, overall survival; PFS, progression-free survival; RT-qPCR, reverse transcription quantitative polymerase chain reaction

Key words: epithelial ovarian cancer, chloride intracellular channel 1 , prognosis

\section{Introduction}

Ovarian cancer is a lethal gynecological malignancy, the incidence of which is gradually increasing (1). Notably, survival rates differ depending on the clinical stage at diagnosis. In particular, the 5-year survival rate for patients at all stages of ovarian cancer is estimated to be $40 \%$ following surgery and paclitaxel/cisplatin chemotherapy. This rate increases to $70 \%$ if patients are diagnosed at an early stage $(2,3)$, indicating the importance of early diagnosis. However, only $15 \%$ patients are diagnosed at an early stage of ovarian cancer, whereas approximately $80 \%$ cases are diagnosed at an advanced stage (stage III or IV) with extensive abdominal dissemination or distant metastasis (4).

Epithelial ovarian cancer, which constitutes 70-90\% of all ovarian cancers, represents the predominant pathological type of this disease (5). The biomarker most widely used to monitor epithelial ovarian cancer is serum cancer antigen 125 (CA 125), although this marker exhibits limited sensitivity and specificity in early detection of ovarian cancer $(2,6,7)$. A large randomized controlled trial using a combination of serum CA 125 measurement and ultrasonography for screening ovarian cancer in asymptomatic women showed no benefit in terms of decreased mortality (8). It appears that the only population that may benefit from such ovarian cancer screening may be women with BRCAl and $B R C A 2$ mutations with or without a family history of ovarian cancer $(9,10)$. Thus, reliable methods for screening specific and sensitive markers for early detection of ovarian cancer are required.

Chloride intracellular channel 1 (CLIC1) was first described in the human mononuclear cell line U937 by Valenzuela et al in 1997 (11). Since its discovery, CLIC1 has been identified to have a role in various fundamental biological processes, including maintenance of cell volume, ion homeostasis, trans-epithelial transport, and $\mathrm{pH}$ regulation. Studies have shown that CLIC1 regulates the cell cycle, as well as cell proliferation, apoptosis, and differentiation $(12,13)$. Recently, CLIC1 was shown to be upregulated in gastric, pancreatic, and liver cancers (14-16).

However, although CLIC1 has been associated with several cancers, its clinical significance in ovarian cancer has not yet been determined. In this study, we investigated CLIC1 
expression in subjects with ovarian cancer and in healthy controls to elucidate the association between CLIC1 levels and pathological features in patients with epithelial ovarian cancer. All cases were followed-up to assess the prognostic value of CLIC1 based on progression-free survival (PFS) and overall survival (OS).

\section{Materials and methods}

Patients and tissues. Clinical samples were obtained between 2007 and 2016 from patients undergoing surgery in Beijing Chao-Yang Hospital, Capital Medical University. The study protocol was approved by the Ethics Committee Board of Beijing Chao-Yang Hospital, and informed consent was obtained from all participating patients. In total, 266 patients with epithelial ovarian cancer were included in this study. All tissues were formalin-fixed and embedded in paraffin blocks. All diagnoses were confirmed by pathological examination. Patients with additional confirmed malignant gynecological tumors or those who received surgical treatments for ovarian cancer were also excluded. Patients with insufficient data or those who died of unknown causes were excluded from follow-up (Fig. 1).

Clinicopathological data, including age, pathological type, histological grade, tumor size, serum cancer antigen (CA) 125 level and International Federation of Gynecology and Obstetrics (FIGO) stage, were retrospectively reviewed. Patient age ranged from 35 to 74 years, with a mean age of $54.3 \pm 9.2$ years. Among the 266 patients with epithelial ovarian cancer, 188 (70.7\%) were diagnosed with serous adenocarcinoma, $26(9.8 \%)$ had endometrioid adenocarcinoma, $22(8.3 \%)$ had clear cell adenocarcinoma, and $30(11.2 \%)$ had mucous adenocarcinoma. Of these cases, 72 were in stages I-II, whereas 194 were in stages III-IV. All cases were followed-up and assessed in outpatient clinics or over the telephone at 3-month intervals for the first two years, 4-6 month intervals for the following three years and at yearly intervals if the patients were disease-free for five years. All patients were followed-up until end of 2017 or until death, with an average follow-up of 4.4 years.

Reverse transcription-quantitative polymerase chain reaction $(R T-q P C R)$. Total tissue RNA was extracted using TRIzol (Takara Bio, Inc., Otsu, Japan) according to the manufacturer's instructions. The RT reaction was conducted in a $20-\mu 1$ reaction volume using a One Step PrimeScript RT-PCR Kit (Takara Bio, Inc.) on an ABI Prism 7500 Real-Time PCR system (Applied Biosystems; Thermo Fisher Scientific, Inc., Waltham, MA, USA). The RT reaction was performed at $42^{\circ} \mathrm{C}$ for $5 \mathrm{~min}$ and $95^{\circ} \mathrm{C}$ for $10 \mathrm{sec}$. The PCR amplification was performed at $95^{\circ} \mathrm{C}$ for $5 \mathrm{sec}$ and $60^{\circ} \mathrm{C}$ for $34 \mathrm{sec}$ for 40 consecutive cycles. Human $\beta$-actin was used as an endogenous control. Relative mRNA levels were calculated using the $2^{-\Delta \Delta \mathrm{Cq}}$ method (17). The following primers were used: CLIC1 forward, 5'-GTT GACACCAAAAGGCGGAC-3', and reverse, 5'-GGGCTT TCAGGAGTCCCTTC-3'; $\beta$-actin forward, 5'-CCTGGCACC CAGCACAAT-3', and reverse: 5'-GCTGATCCACATCTG CTGGAA-3'. All primers were synthesized by GenePharma (Shanghai, China).

Western blot analysis. Western blotting was performed on cancer and healthy samples. The tissues were stored at $-80^{\circ} \mathrm{C}$.
Total protein was extracted by ristocetin-induced platelet aggregation (Beyotime Institute of Biotechnology, Jiangsu, China) in the presence of phosphatase and protease inhibitors according to the manufacturer's instructions (Beijing Solarbio Science \& Technology Co., Ltd., Beijing, China). Protein concentrations were determined using a bicinchoninic acid (BCA) protein assay kit according to the manufacturer's instructions (Boster Biological Technology Co., Ltd., Wuhan, China). Thirty micrograms of protein per lane were separated by $10 \%$ sodium dodecyl sulfate polyacrylamide gel electrophoresis and transferred to $0.45 \mu \mathrm{m}$ nitrocellulose filter membrane (EMD Millipore, Billerica, MA, USA), followed by blocking with skim milk at room temperature for 2 h. Subsequently, the membranes were incubated with mouse anti-human CLIC1 antibody (ab77214; 1:750 dilution; Abcam, Cambridge, $\mathrm{UK})$ or mouse anti-human $\beta$-actin (1:2,000 dilution; Boster Biological Technology, Pleasanton, CA, USA) overnight at $4^{\circ} \mathrm{C}$. The membranes were incubated with horseradish peroxidase-conjugated goat anti-mouse antibody (1:2,500 dilution; Boster Biological Technology) for $1 \mathrm{~h}$ and then subjected to semi-quantification by electrochemiluminescence (Bio-Rad Laboratories, Inc., Hercules, CA, USA).

Immunohistochemical staining of tissue microarrays. CLIC1 levels in epithelial ovarian cancer tissues were evaluated by immunohistochemical staining of issue microarray samples. All specimens were obtained from the Pathology Center of the Beijing Chao-Yang Hospital, Capital Medical University. A total of 36 ovarian samples were included in the study as healthy controls. Briefly, formalin-fixed and paraffin-embedded samples were cut into $4-\mu \mathrm{m}$-thick sections and placed on slides. The tissues were dewaxed by baking at $70^{\circ} \mathrm{C}$ for $60 \mathrm{~min}$. Sections were deparaffinized with xylene (10 min each for 3 times) and rehydrated in graded alcohol (5 min each). For antigen retrieval, tissue sections were heated in an autoclave with citrate buffer solution (10 mM, pH 6.0) for $20 \mathrm{~min}$. Blocking of endogenous peroxidase activity was performed using 3\% hydrogen peroxide, followed by rinsing thrice in phosphate-buffered saline. The slides were incubated in normal goat serum at room temperature for $60 \mathrm{~min}$ and then labeled with mouse anti-human CLIC1 antibody (ab77214; Abcam) at a 1:200 dilution overnight at $4^{\circ} \mathrm{C}$. A horseradish peroxidase-conjugated goat anti-mouse antibody (Zhongshan Golden Bridge Biotechnology, Beijing, China) was added as the secondary antibody for $60 \mathrm{~min}$ at room temperature, followed by diaminobenzidine coloration, hematoxylin counterstaining, and neutral resin sealing. Ovarian cancer cell line A2780 was used as the positive control. Mouse nonimmune serum and phosphate-buffered saline with no visible staining were used as the negative and blank controls, respectively.

Evaluation of immunohistochemical staining. All samples were independently examined and evaluated by two senior pathologists who had no knowledge of patient information. Positivity was determined by the presence of yellow or brown staining in the cytoplasmic membrane or nucleus. For semi-quantification, an H-score-based assessment that combined both the percentage $(0-100 \%)$ of stained cells and staining intensity $(0$, none; 1 , weak; 2 , moderate; 3 , strong) 


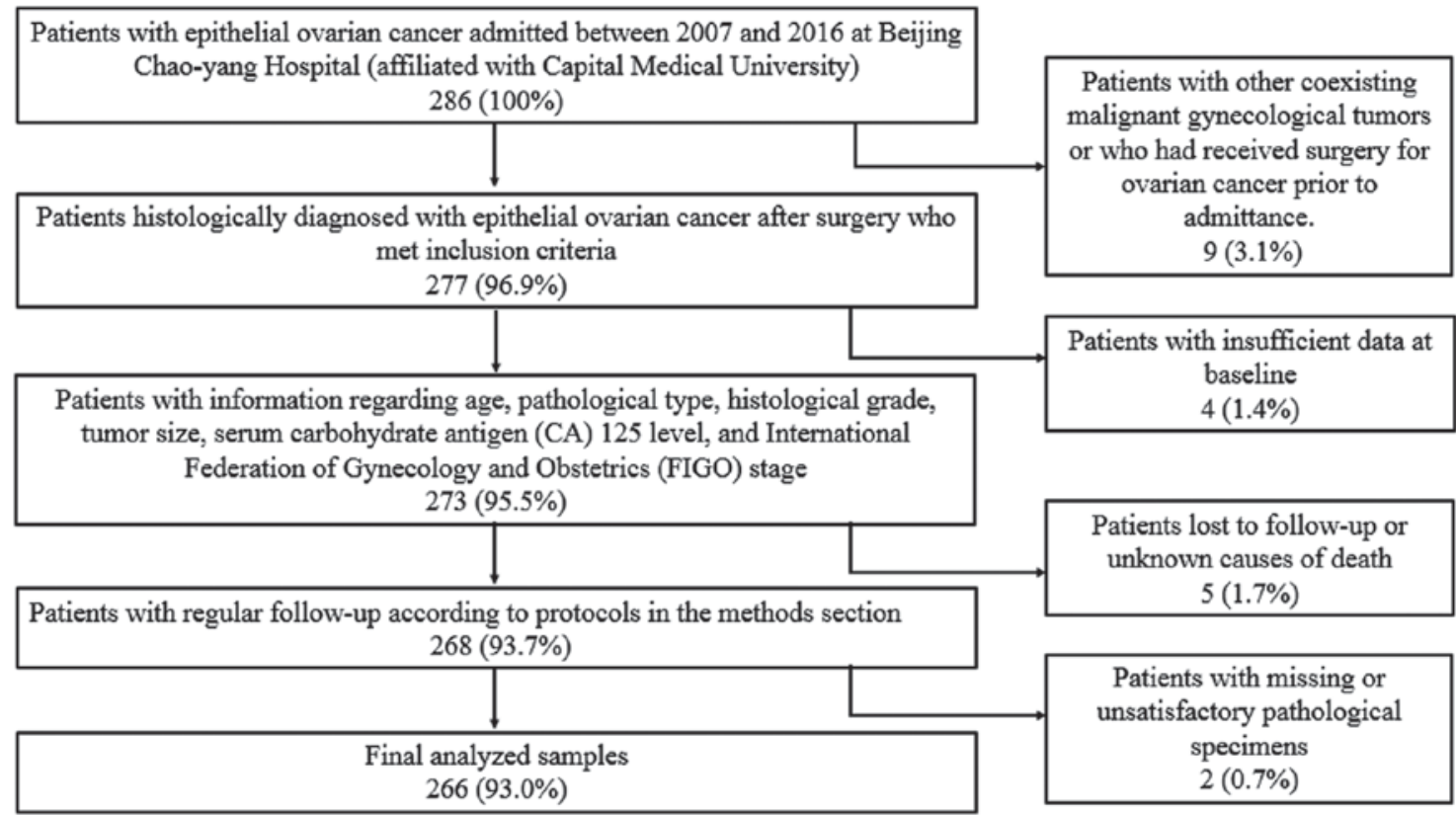

Figure 1. Patient enrollment flowchart.
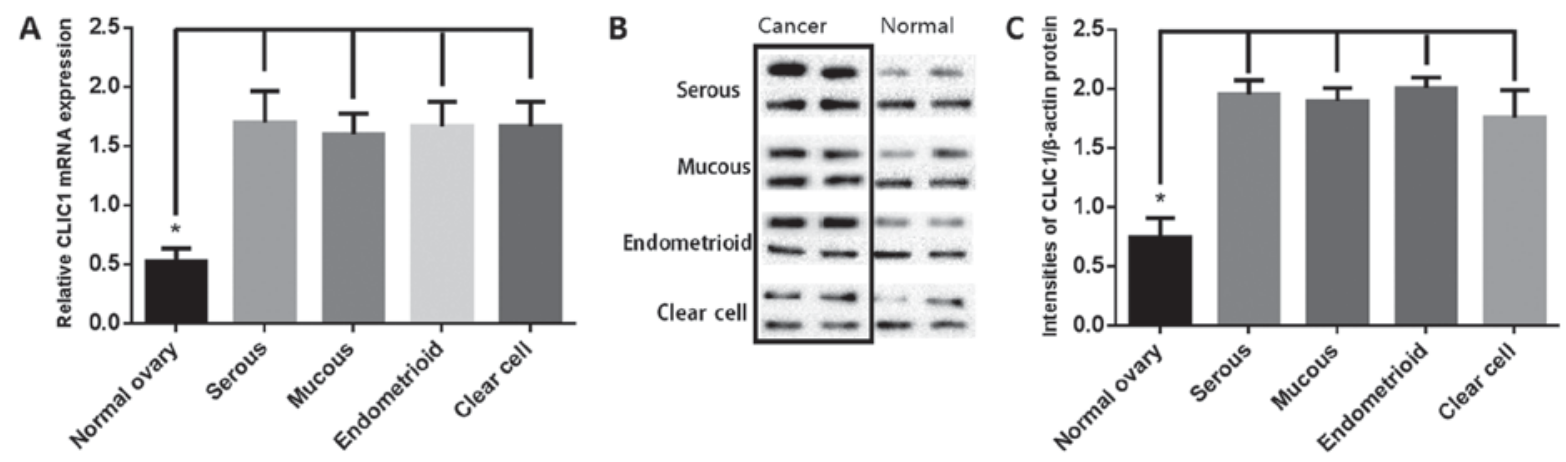

Figure 2. Reverse transcription-quantitative polymerase chain reaction and western blotting analysis of chloride intracellular channel 1 (CLIC1) expression levels in four types of epithelial ovarian cancer tissues and paired healthy ovarian tissues. (A) Relative CLIC1 mRNA level in ovarian cancer samples was higher than that in matched healthy samples. There were no differences between different histological tumor types. The mRNA levels were calculated by $2^{-\triangle \Delta C q}$ method. Human $\beta$-actin was used as the internal control. Data are presented as mean \pm SD. "P<0.01. (B) Representative images of western blotting from 4 groups of cancer and healthy samples are shown. Human $\beta$-actin was used as a loading control. (C) Signal intensities of the western blot bands were analyzed using Image J $1.49 \mathrm{v}$. There was no difference in $\beta$-actin between healthy and tumorous tissues. CLIC1 protein level was significantly higher in tumor tissues than in healthy controls. " $\mathrm{P}<0.01$.

was performed using the following formula: H-score $=\Sigma$ percentage $\mathrm{x}$ intensity. A digital pathological section scanner KF-PRO-005 (KFBIO, Ningbo, China) was used to score tissues to avoid subjectivity.

Statistical analysis. Statistical analyses were conducted using the SPSS statistical package for Windows (version 21.0; IBM Corp., Armonk, NY, USA). Chi-square or Kruskal-Wallis tests were performed to compare categorical variables. Associations between clinical features and CLIC1 expression were examined by Student's t-test and one-way analysis of variance with Fisher's Least Significant Difference post hoc test. Univariate survival analyses were estimated using the Kaplan-Meier method and compared by log-rank testing. Cox regression models were used in multivariate analyses. All experiments were repeated thrice. A two-sided $\mathrm{P}<0.05$ was considered to indicate a statistically significant difference.

\section{Results}

Western blotting and RT-qPCR analyses of CLIC1 expression. CLIC1 mRNA and protein levels were analyzed by RT-qPCR and western blotting of 16 each of ovarian cancer tissues (four each of serous, mucous, endometrioid, and clear cell adenocarcinoma) and paired healthy ovarian tissues. As shown in Fig. 2A, CLIC1 transcript levels were significantly higher in cancer samples than in healthy ovarian tissues. Western blotting was performed to determine the CLIC1 levels (Fig. 2B). Results of semi-quantitative western blotting indicated that CLIC1 levels in ovarian cancer tissues was significantly higher than that in healthy ovarian tissues, which is consistent with the RT-qPCR results (Fig. 2C).

Immunohistochemical assessment of CLIC1 levels in epithelial ovarian cancer. Immunohistochemical staining showed 
Table I. Association between CLIC1 expression and clinicopathological features in patients with epithelial ovarian cancer.

\begin{tabular}{|c|c|c|c|}
\hline Feature & $\begin{array}{c}\text { No. of } \\
\text { cases }\end{array}$ & H-score & P-value \\
\hline Age (years) & & & 0.692 \\
\hline$<55$ & 139 & $177.8 \pm 21.3$ & \\
\hline$\geq 55$ & 127 & $178.9 \pm 22.4$ & \\
\hline Tumor diameter $(\mathrm{cm})$ & & & 0.397 \\
\hline$<5$ & 81 & $180.1 \pm 22.3$ & \\
\hline$\geq 5$ & 185 & $177.6 \pm 21.6$ & \\
\hline Histological type & & & 0.085 \\
\hline Serous & 188 & $180.5 \pm 20.9$ & \\
\hline Mucus & 30 & $174.7 \pm 26.5$ & \\
\hline Endometrioid & 26 & $172.5 \pm 20.7$ & \\
\hline Clear cell & 22 & $171.7 \pm 22.6$ & \\
\hline Tumor Grade & & & $<0.001$ \\
\hline Low & 28 & $162.6 \pm 31.9$ & \\
\hline Moderate & 73 & $177.6 \pm 17.8$ & \\
\hline High & 165 & $181.3 \pm 20.3$ & \\
\hline Serum CA-125 level (U/ml) & & & 0.979 \\
\hline$<35$ & 78 & $178.3 \pm 20.2$ & \\
\hline$\geq 35$ & 188 & $178.4 \pm 22.5$ & \\
\hline FIGO stage & & & 0.539 \\
\hline Early (I-II) & 72 & $177.0 \pm 23.1$ & \\
\hline Advanced (III-IV) & 194 & $178.8 \pm 21.4$ & \\
\hline Cisplatin resistance & & & $<0.001$ \\
\hline Yes & 90 & $185.0 \pm 20.1$ & \\
\hline No & 176 & $172.9 \pm 22.0$ & \\
\hline
\end{tabular}

CA, cancer antigen; CLIC1, chloride intracellular channel 1; FIGO, International Federation of Gynecology and Obstetrics.

negative staining in healthy ovarian tissues and paracancerous tissues, while positive CLIC1 signals were observed in the four types of epithelial ovarian cancer cells (Fig. 3). CLIC1 staining signals were significantly higher in the 266 samples from patients with epithelial ovarian cancer $(\mathrm{P}<0.001)$ than that observed in healthy controls. There was no difference between different pathological types.

Correlations between CLIC1 expression and clinicopathological features. The relationship between CLIC1 expression and clinicopathological parameters in patients with epithelial ovarian cancer, including age, pathological type, histological grade, tumor size, serum CA-125 levels, FIGO stage, and cisplatin resistance, were evaluated. However, there were no significant associations between CLIC1 levels and patient age, tumor type, FIGO stage, or cisplatin resistance in patients with epithelial ovarian cancer (Table I), wherein cisplatin resistance was associated with a PFS interval less than six months after cisplatin therapy. For the histological grade, a markedly higher level of CLIC1 was observed in patients with high-grade tumors than those with low-grade tumors. Furthermore,
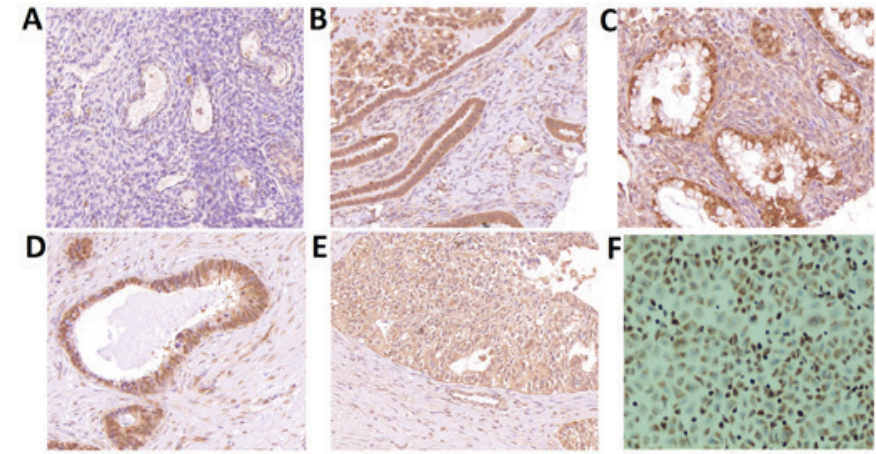

Figure 3. Immunohistochemical assessment of chloride intracellular channel 1 (CLIC1) expression. (A) CLIC1 protein was not present in healthy ovarian tissues. (B) CLIC1 protein was expressed in both the nuclei and plasma membrane of mucous adenocarcinoma samples. (C) Cytoplasmic staining was found in serous adenocarcinoma cells. (D) Nuclear staining was observed in endometrioid adenocarcinoma cells. (E) CLIC1 protein was located on cell membrane of clear cell adenocarcinoma cells. (F) Ovarian cancer cell line A2780 was used as a positive control. Original magnification, x200.

patients with cisplatin resistance had higher levels of CLIC1 than those sensitive to cisplatin therapy $(\mathrm{P}<0.001)$.

Relationship between CLIC1 expression and prognosis. In subsequent analysis, we determined the relationship between CLIC1 expression and patient prognosis using a Kaplan-Meier survival curve and Cox proportional hazards model. During the follow-up period, $41.4 \%$ (110/266) patients died, whereas $28.6 \%$ (76/266) remained progression-free. The shortest PFS interval was 1 month, and the longest was 118 months, with a mean PFS period of $21.4 \pm 15.7$ months. In the multivariate analysis, we identified FIGO stage $(\mathrm{P}=0.011)$ and CLIC1 expression $(\mathrm{P}=0.002)$ as independent risk factors affecting $\mathrm{OS}$ in all cases (Fig. 4A). Patients who had higher CLIC1 levels also had poorer OS. Investigation of factors affecting PFS by Cox regression analysis showed that tumor grade $(\mathrm{P}=0.030)$, FIGO stage $(\mathrm{P}=0.011)$, CLIC1 expression $(\mathrm{P}=0.006)$, and cisplatin resistance $(\mathrm{P}=0.009)$ were significant independent prognostic factors (Fig. 4B). In the univariate analysis using Kaplan-Meier survival curves, higher CLIC1 levels led to poorer prognosis in terms of both PFS $(\mathrm{P}=0.034)$ and $\mathrm{OS}(\mathrm{P}=0.014$; Fig. 5), which is consistent with the results of the Cox model. These results suggested that CLIC1 overexpression is associated with poor prognosis of patients with epithelial ovarian cancer.

\section{Discussion}

In the current study, we evaluated the ability of CLIC1 to serve as a biomarker for epithelial ovarian cancer. Results of RT-qPCR and western blotting showed that CLIC1 mRNA and protein levels were significantly upregulated in ovarian cancer tissues than in healthy controls. We also evaluated the expression of CLIC1 in 266 patients with ovarian cancer by immunohistochemical staining, which showed that CLIC1 level was significantly higher in ovarian cancer tissues than in healthy ovarian tissues.

All seven members of the CLIC protein family (CLIC1-6 and p64) are components of mammalian ion channels (18-21) with highly conserved carboxyl termini. As a member of the CLIC family, CLIC1 is widely expressed in various species 
A

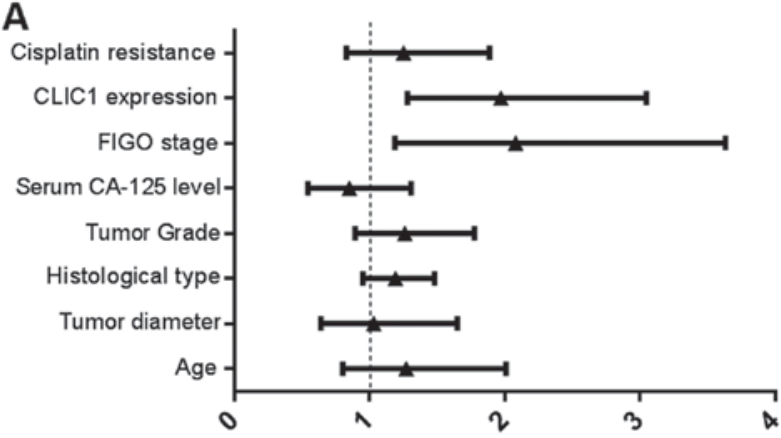

B

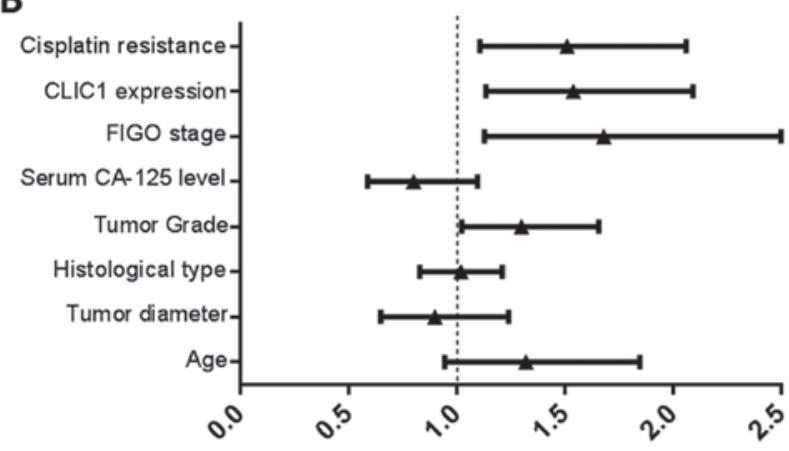

Hazard ratio $(95 \% \mathrm{Cl}) \quad \mathrm{P}$

$1.25(0.827-1.886) \quad 0.291$

$1.97(1.279-3.046) \quad 0.002^{*}$

$2.08(1.186-3.631) \quad 0.011^{*}$

$0.85(0.543-1.305) \quad 0.442$

$1.26(0.893-1.774) \quad 0.190$

$1.19(0.951-1.478) \quad 0.131$

$1.03(0.640-1.648) \quad 0.912$

$1.27(0.801-2.088) \quad 0.312$

Hazard ratio $(95 \% \mathrm{Cl}) \quad \mathrm{P}$

$1.51(1.107-2.060) \quad 0.009^{*}$

$1.54(1.135-2.092) \quad 0.006^{*}$

$1.68(1.128-2.499) \quad 0.011^{*}$

$0.80(0.588-1.096) \quad 0.166$

$1.30(1.024-1.656) \quad 0.030^{*}$

$1.02(0.830-1.210) \quad 0.986$

$0.90(0.649-1.240) \quad 0.509$

$1.32(0.945-1.846) \quad 0.104$

Figure 4. Multivariate analysis independent risk factors affecting overall survival (OS) and progression-free survival (PFS). (A) International Federation of Gynecology and Obstetrics (FIGO) stage and chloride intracellular channel 1 (CLIC1) expression were identified as independent risk factors affecting OS. (B) Tumor grade, FIGO stage, CLIC1 expression, and cisplatin resistance were significant independent prognostic factors for PFS. "P<0.05.

A

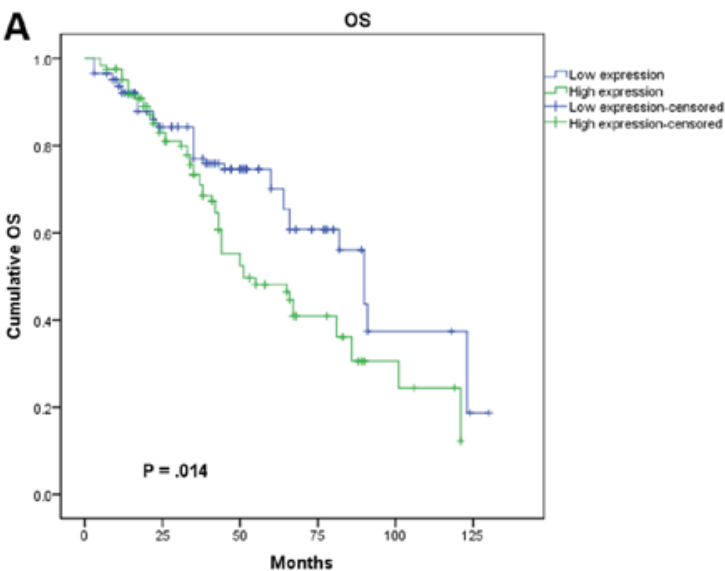

B

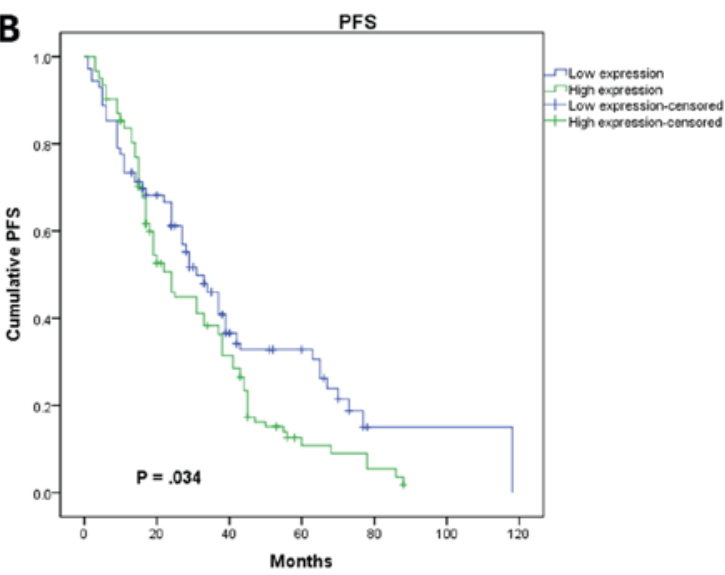

Figure 5. Kaplan-Meier survival curves analysis of association between chloride intracellular channel 1 (CLIC1) expression and prognosis of patients with epithelial ovarian cancer. (A) CLIC1 expression was strongly associated with shorter overall survival (OS) in epithelial ovarian cancer patients (P=0.014). (B) CLIC1 expression was significantly related with shorter progression-free survival (PFS) in epithelial ovarian cancer patients $(\mathrm{P}=0.034)$.

and exists in both plasma-soluble and membrane-binding forms. Notably, CLIC1 can auto-transit from the cytoplasm to the plasma membrane without transport vesicles during cell proliferation (22). A study in Chinese hamster ovary cells showed that chloride conductance differed based on the cell cycle phase and that CLIC1 largely relocated to plasma membranes in the G2/M phase. The application of a chloride ion channel blocker led to the arrest of cells in the G2/M phase, indicating that CLIC1 was involved in cell cycle regulation (12). Peretti et al (23) and Gurski et al (24) showed that CLIC1 was strongly overexpressed in certain tumor types and that it translocated from the cytoplasm to the membrane to act as a signal transducer during cell cycle progression in pathological conditions and neoplastic transformation, indicating that it might be a novel therapeutic target for cancer.

CLIC1 regulates anti-apoptosis signaling pathways and cellular transformation (25). Further analyses of the relationship between CLIC1 expression and clinical features showed that CLIC1 expression was more intense in poorly differentiated than in well-differentiated ovarian carcinoma. In addition, we showed in a previous study that CLICl knockdown slowed the growth and proliferation of ovarian cancer cells (A2780) via cell cycle arrest in the G1 phase in vitro, suggesting that upregulated CLIC1 promoted cell transformation (26). Similar results were also reported by Li et al (15), who observed that CLIC1 contributed to the proliferation of Hca-F hepatic 
carcinoma cells and overexpression of CLIC1 modulated cell division and resulted in cellular transformation. Furthermore, we observed that patients with lower CLIC1 levels showed increased sensitivity to cisplatin treatment in 266 patients with ovarian cancer. This was possibly because CLIC1 participates in the regulation of anti-apoptosis signaling pathways in response to chemotherapy. Similarly, Kang observed that application of a chloride channel blocker to a human glioblastoma multiforme cell line promoted the killing effect of chemotherapeutic drugs and accelerate cell apoptosis.

Several studies have also indicated that CLIC1 is involved in tumor angiogenesis, possibly by regulating the expression of integrins on endothelial cell surfaces (27). Integrins are important surface molecules that mediate cell migration and invasion, cellular attachment, adhesion to the extracellular matrix, intracellular signaling, and cell growth and proliferation (28). Tung and Kitajewski (29) reported that CLICI knockdown increased the expression of integrin subtypes on endothelial cell surfaces, leading to a noticeably decreased density of capillary sprouting and branching compared to that observed in the control groups, indicating that lower levels of CLIC1 can moderate tumor cell migration and capillary network formation by regulating the expression of endothelial integrins. High-grade cancer exhibits strong invasion and migration ability, mainly via blood and lymphatic metastases. In the present study, we observed that patients with high-grade ovarian cancer had higher CLIC1 levels than patients with low-grade tumors. Shorter PFS and OS were expected in patients harboring such tumors.

This study has some limitations. There could have been selection bias owing to the retrospective nature of the analysis. Since our hospital is a tertiary referral institution, it is possible that patients with serious conditions are more likely to be admitted to our center because of referral bias. Their outcomes may differ from those of mild cases who were not transferred to our center.

Taken together, our observations indicate that the CLIC1 is significantly overexpressed in epithelial ovarian cancer tissues. Overexpressed CLIC1 may promote malignant transformation and increase cisplatin resistance, indicating a poor prognosis for patients with ovarian cancer.

\section{Acknowledgements}

Not applicable.

\section{Funding}

This study was supported by International Science and Technology Cooperation Program of China (grant no. 2012DFR30490).

\section{Availability of data and materials}

The datasets used and/or analyzed during the current study are available from the corresponding author on reasonable request.

\section{Authors' contributions}

WY, CL and HD conceived and designed the study; WY, $\mathrm{RC}, \mathrm{HQ}$ and $\mathrm{CL}$ acquired, analyzed and interpreted the raw data; HD and ZZ revised it critically for important intellectual content. ZZ also obtained the raw data and gave final approval of the version to be published.

\section{Ethics approval and consent to participate}

The study protocol was approved by the Ethics Committee Board of Beijing Chao-Yang Hospital (reference no. 2008L01498), and informed consent was obtained from all participating patients.

\section{Consent for publication}

The patient, or parent, guardian or next of kin provided written informed consent for the publication of any associated data and accompanying images.

\section{Competing interests}

The authors declare that they have no competing interests.

\section{References}

1. Webb PM and Jordan SJ: Epidemiology of epithelial ovarian cancer. Best Prac Res Clin Obste Gynaecol 41: 3-14, 2017.

2. Mills K and Fuh K: Recent advances in understanding, diagnosing, and treating ovarian cancer. F1000Res 6: 84, 2017.

3. Oronsky B, Ray CM, Spira AI, Trepel JB, Carter CA and Cottrill HM: A brief review of the management of platinum-resistant-platinum-refractory ovarian cancer. Med Oncol 34: 103, 2017.

4. Siegel RL, Miller KD and Jemal A: Cancer statistics, 2016. CA Cancer J Clin 66: 7-30, 2016.

5. Jessmon P, Boulanger T,Zhou W and Patwardhan P: Epidemiology and treatment patterns of epithelial ovarian cancer. Expert Rev Anticancer Ther 17: 427-437, 2017.

6. Yang WL, Lu Z and Bast RC Jr: The role of biomarkers in the management of epithelial ovarian cancer. Expert Rev Mol Diagn 17: 577-591, 2017.

7. Ebell MH, Culp MB and Radke TJ: A systematic review of symptoms for the diagnosis of ovarian cancer. Am J Prev Med 50: 384-394, 2016.

8. Buys SS, Partridge E, Black A, Johnson CC, Lamerato L, Isaacs C, Reding DJ, Greenlee RT, Yokochi LA, Kessel B, et al: Effect of screening on ovarian cancer mortality: The prostate, lung, colorectal and ovarian (PLCO) cancer screening randomized controlled trial. JAMA 305: 2295-2303, 2011.

9. Futreal PA, Liu Q, Shattuck-Eidens D, Cochran C, Harshman K, Tavtigian S, Bennett LM, Haugen-Strano A, Swensen J, Miki Y, et al: BRCA1 mutations in primary breast and ovarian carcinomas. Science 266: 120-122, 1994.

10. Bayraktar S and Arun B: BRCA mutation genetic testing implications in the United States. Breast 31: 224-232, 2017.

11. Valenzuela SM, Martin DK, Por SB, Robbins JM, Warton K, Bootcov MR, Schofield PR, Campbell TJ and Breit SN: Molecular cloning and expression of a chloride ion channel of cell nuclei. J Biol Chem 272: 12575-12582, 1997.

12. Valenzuela SM, Mazzanti M, Tonini R, Qiu MR, Warton K, Musgrove EA, Campbell TJ and Breit SN: The nuclear chloride ion channel NCC27 is involved in regulation of the cell cycle. J Physiol 529: 541-552, 2000.

13. Lu J, Dong Q, Zhang B, Wang X, Ye B, Zhang F, Song X, Gao G, $\mathrm{Mu}$ J, Wang Z, et al: Chloride intracellular channel 1 (CLIC1) is activated and functions as an oncogene in pancreatic cancer. Med Oncol 32: 616, 2015.

14. Jia N, Dong S, Zhao G, Gao H, Li X and Zhang H: CLIC1 overexpression is associated with poor prognosis in pancreatic ductal adenocarcinomas. J Cancer Res Ther 12: 892-896, 2016.

15. Li RK, Zhang J, Zhang YH, Li ML, Wang M and Tang JW: Chloride intracellular channel 1 is an important factor in the lymphatic metastasis of hepatocarcinoma. Biomed Pharmacother 66: 167-172, 2012. 
16. Ma PF, Chen JQ, Wang Z, Liu JL and Li BP: Function of chloride intracellular channel 1 in gastric cancer cells. World J Gastroenterol 18: 3070-3080, 2012.

17. Livak KJ and Schmittgen TD: Analysis of relative gene expression data using real-time quantitative PCR and the 2(-Delta Delta C(T)) method. Methods 25: 402-408, 2001.

18. Averaimo S, Milton RH, Duchen MR and Mazzanti M: Chloride intracellular channel 1 (CLIC1): Sensor and effector during oxidative stress. FEBS Lett 584: 2076-2084, 2010.

19. Ulmasov B, Bruno J, Woost PG and Edwards JC: Tissue and subcellular distribution of CLIC1. BMC Cell Biol 8: 8, 2007.

20. Littler DR, Harrop SJ, Brown LJ, Pankhurst GJ, Mynott AV, Luciani P, Mandyam RA, Mazzanti M, Tanda S, Berryman MA, et al: Comparison of vertebrate and invertebrate CLIC proteins: The crystal structures of Caenorhabditis elegans EXC-4 and Drosophila melanogaster DmCLIC. Proteins 71: 364-378, 2008.

21. Ashley RH: Challenging accepted ion channel biology: p64 and the CLIC family of putative intracellular anion channel proteins (Review). Mol Membr Biol 20: 1-11, 2003.

22. Goodchild SC, Howell MW, Cordina NM, Littler DR, Breit SN, Curmi PM and Brown LJ: Oxidation promotes insertion of the CLIC1 chloride intracellular channel into the membrane. Eur Biophys J 39: 129-138, 2009.

23. Peretti M, Angelini M, Savalli N, Florio T, Yuspa SH and Mazzanti M: Chloride channels in cancer: Focus on chloride intracellular channel 1 and 4 (CLIC1 AND CLIC4) proteins in tumor development and as novel therapeutic targets. Biochim Biophys Acta 1848: 2523-2531, 2015.
24. Gurski LA, Knowles LM, Basse PH, Maranchie JK, Watkins SC and Pilch J: Relocation of CLIC1 promotes tumor cell invasion and colonization of fibrin. Mol Cancer Res 13: 273-280, 2015.

25. Kang MK and Kang SK: Pharmacologic blockade of chloride channel synergistically enhances apoptosis of chemotherapeutic drug-resistant cancer stem cells. Biochem Biophys Res Commun 373: 539-544, 2008.

26. Qu H, Chen Y, Cao G, Liu C, Xu J, Deng H and Zhang Z: Identification and validation of differentially expressed proteins in epithelial ovarian cancers using quantitative proteomics. Oncotarget 7: 83187-83199, 2016.

27. Biasiotta A, D'Arcangelo D, Passarelli F, Nicodemi EM and Facchiano A: Ion channels expression and function are strongly modified in solid tumors and vascular malformations. J Trans Med 14: 285, 2016.

28. Hood JD and Cheresh DA: Role of integrins in cell invasion and migration. Nat Rev Cancer 2: 91-100, 2002.

29. Tung JJ and Kitajewski J: Chloride intracellular channel 1 functions in endothelial cell growth and migration. J Angiogenes Res 2: 23, 2010.

(i) (9) This work is licensed under a Creative Common

EY No NO Attribution-NonCommercial-NoDerivatives 4.0 International (CC BY-NC-ND 4.0) License. 